\title{
Comprender las dimensiones del sistema digital
}

Recibido: 27 de julio de 2018

Aceptado: 17 de agosto de 2018

Publicado: 28 de noviembre de 2018
Octavio Islas

octavio.islas@uhemisferios.edu.ec

Amaia Arribas

amayaa@uhemisferios.edu.ec

Universidad de Los Hemisferios (Ecuador)

Resumen: El objetivo del artículo es destacar las dimensiones del sistema digital. En ese sentido, presentamos cifras relativas a la penetración mundial de Internet y analizamos la penetración de Internet en el continente americano. Asimismo, centramos nuestra atención en las redes sociales, y presentamos las cifras más significativas de Facebook. Finalmente referimos el estudio del World Economic Forum, indispensable para comprender la importancia de Internet y las redes sociales en el desarrollo de los sistemas de telecomunicaciones.

Palabras clave: Internet, penetración de Internet, redes sociales, telecomunicaciones.

Abstract: Our objective is to highlight the dimensions of the digital system. To do it, we present figures related to the global penetration of the Internet and analyze the penetration of the Internet in the Americas. Then we focus our attention on social networks, and present the most significant Facebook stats. Finally, we refer the studies of the World Economic Forum, which are essential for understanding the importance of the Internet and social networks in the development of telecommunications systems.

Key words: Internet, Internet Penetration, Social Media, Telecommunications. 


\section{La penetración de Internet en el mundo}

Internet es el complejo medio de comunicación que efectivamente materializó el concepto "aldea global", que en la década de 1960 introdujo Marshall McLuhan'. En el futuro, el desarrollo de los nuevos medios de comunicación fundamentalmente dependerá de Internet y de tecnologías derivadas. Por ello es tan importante comprender las dimensiones del sistema digital y reconocer las dimensiones de Internet.

En este apartado recurrimos a tres autorizadas fuentes de información: Internet World Stats, IWS (2017), We are Social \& Hootsuite $(2017,2018)$ y la International Telecommunications Union, UIT (2017) para reconocer las dimensiones de Internet, el gran sistema digital que hoy representa la columna vertebral de todos los sistemas de telecomunicaciones en el planeta.

De acuerdo con información de la Internet World Stats (IWS), el 31 de diciembre de 2017, la población mundial fue estimada en 7634758428 personas, de las cuales, 4156932140 son usuarios de Internet. La penetración mundial de Internet fue estimada en 54.4\%. Para los propósitos de sus estudios, la IWS (2017) divide al mundo en siete regiones: África, Asia, Europa, América Latina y Caribe, Medio Oriente, Norteamérica, Oceanía/Australia. En la tabla 1 podremos reconocer la población estimada en cada región, el número de usuarios de Internet y la penetración de Internet por región.

Tabla 1: Población y usuarios de Internet en el mundo (diciembre de 2017).

\begin{tabular}{|l|r|r|c|}
\hline \multicolumn{1}{|c|}{ Región } & Población estimada & Internautas & $\begin{array}{c}\text { Penetración de Internet } \\
\text { en la población }\end{array}$ \\
\hline África & 1287914329 & 453329534 & $35.2 \%$ \\
\hline Asia & 4207588157 & 2023630194 & $48.1 \%$ \\
\hline Europa & 827650849 & 704833752 & $85.2 \%$ \\
\hline $\begin{array}{l}\text { América Latina } \\
\text { y el Caribe }\end{array}$ & 652047996 & 437001277 & $67 \%$ \\
\hline Medio Oriente & 254438981 & 164037259 & $64.5 \%$ \\
\hline América del Norte & 363844662 & 345660847 & $95 \%$ \\
\hline Oceanía/Australia & 41273454 & 28439277 & $68.9 \%$ \\
\hline Totales & $\mathbf{7 6 3 4 7 5 8 4 2 8}$ & $\mathbf{4 1 5 6 9 3 2 1 4 0}$ & $\mathbf{5 4 . 4 \%}$ \\
\hline
\end{tabular}

Fuente: Internet World Stats (2017).

1 Término que acuñó Marshall McLuhan en su tercer libro La Galaxia Gutenberg. La génesis del hombre tipográfico (1962). Además, nueve años después de su muerte (1980) fue publicado el libro The global village: Transformations in world life and media in the 21st century, en el cual McLuhan figura como autor principal. 
En África se registra la menor penetración de Internet (35.2\%). Asia concentra la mayor cantidad de habitantes en el mundo (4207 588157) y el mayor número de usuarios de Internet (2 023630 194). Sin embargo, la penetración de Internet (48.1\%) es inferior al promedio mundial $(54.4 \%)$. Asia representa un mercado muy atractivo para todas aquellas empresas e industrias vinculadas con el desarrollo de la economía digital. Europa es la segunda región con el porcentaje más alto de usuarios de Internet (85.2\%).

En América Latina y el Caribe, la penetración de Internet (67\%) es superior al promedio mundial. En Medio Oriente, la penetración de Internet (64.5\%) es inferior a la estimada en América Latina (67\%), pero superior al promedio mundial (54.4\%). América del Norte presenta la penetración de Internet más alta (95\%). Australia-Oceanía es la región con menor población (41 273 454). La penetración de Internet (68.9\%) apenas es superior a la estimada en América Latina y Caribe (67\%).

En sus estudios, We are Social \& Hootsuite (2018) dividen al mundo en cinco bloques: África, América, Asia-Pacífico, Europa, Medio Oriente. En la tabla 2 podremos reconocer la población estimada en cada bloque, el total de usuarios de Internet y la penetración de Internet en la población.

Tabla 2: Población y usuarios de Internet en el mundo (enero de 2018).

\begin{tabular}{|l|r|r|c|}
\hline \multicolumn{1}{|c|}{ Región } & Población estimada & Usuarios de Internet & $\begin{array}{c}\text { Penetración de Internet } \\
\text { en la población }\end{array}$ \\
\hline África & 1272000000 & 435000000 & $34 \%$ \\
\hline América & 1011000000 & 741000000 & $73 \%$ \\
\hline Asia-Pacífico & 4214000000 & 2007000000 & $48 \%$ \\
\hline Europa & 843000000 & 674000000 & $80 \%$ \\
\hline Medio Oriente & 252000000 & 164000000 & $65 \%$ \\
\hline Total & $\mathbf{7 5 9 3 0 0 0 0 0 0}$ & $\mathbf{4 0 2 1 0 0 0 0 0 0}$ & $\mathbf{5 3 \%}$ \\
\hline
\end{tabular}

Fuente: We are Social \& Hootsuite (2018).

La penetración de Internet en África es la más baja (34\%), la cual, además es inferior al promedio mundial (53\%). En Asia-Pacífico se concentra el mayor número de personas (4 214000000 ) y, por supuesto, el mayor número de usuarios de Internet (2 007000000 ). Sin embargo, al igual que África, la penetración de Internet (48\%) es inferior al promedio mundial (53\%). Europa es la región que presenta la más alta penetración de Internet en la población (80\%). Por lo que respecta a Medio Oriente, las cifras que presenta este estudio son muy parecidas a las que ofrece la IWS (2017). En la tabla 3 se ofrece información más detallada sobre la penetración mundial de Internet por regiones: 
Tabla 3: Penetración de Internet en el mundo (enero, 2018).

\begin{tabular}{|c|c|c|}
\hline Región & $\begin{array}{c}\text { Millones de } \\
\text { usuarios }\end{array}$ & $\begin{array}{c}\text { Penetración de } \\
\text { Internet } \\
\text { en la población }\end{array}$ \\
\hline Este de Asia & 947 & $57 \%$ \\
\hline Sur de Asia & 673 & $36 \%$ \\
\hline Sureste de Asia & 380 & $58 \%$ \\
\hline América del Norte & 320 & $88 \%$ \\
\hline Sudamérica & 290 & $68 \%$ \\
\hline Europa del Este & 215 & $74 \%$ \\
\hline Oeste de Asia & 175 & $65 \%$ \\
\hline Europa del Oeste & 175 & $90 \%$ \\
\hline Oeste de África & 147 & $39 \%$ \\
\hline Sur de Europa & 118 & $77 \%$ \\
\hline Este de África & 118 & $27 \%$ \\
\hline Norte de África & 116 & $49 \%$ \\
\hline Centroamérica & 110 & $61 \%$ \\
\hline Norte de Europa & 94 & $98 \%$ \\
\hline Asia Central & 36 & $50 \%$ \\
\hline Sur de África & 34 & $51 \%$ \\
\hline Oceanía & 28 & $69 \%$ \\
\hline Caribe & 21 & $48 \%$ \\
\hline África Central & 20 & $12 \%$ \\
\hline
\end{tabular}

Fuente: We are Social \& Hootsuite (2018).

La región que presenta la más alta penetración de Internet es el Norte de Europa (98\%). Ese dato difiere del que asentó la IWS en su estudio (2017), ubicando a América del Norte como la región que presenta la más alta penetración (95\%). La penetración de Internet en Centroamérica, donde México fue ubicado (61\%), no se encuentra tan rezagada de la penetración de Internet en Sudamérica (68\%). 
En el referido estudio además fueron estimados los internautas móviles (3 722000 000), que representan $49 \%$ de la población mundial. El dato es importante, ya que permite confirmar la migración del mundo digital hacia el imaginario móvil. Además, el estudio destaca que la penetración mundial de Internet creció $7 \%$ en el periodo comprendido entre enero de 2017 y enero de 2018. En ese periodo fue estimada la incorporación de 248 millones de nuevos internautas (We are Social \& Hootsuite, 2017).

En dicho estudio fueron identificadas las diez naciones que presentan la penetración de Internet más alta (tabla 4), consignándose la información relativa a los internautas estimados en cada una de esas naciones y la penetración de Internet.

Tabla 4: Países que presentan la más alta penetración de Internet.

\begin{tabular}{|l|r|c|}
\hline \multicolumn{1}{|c|}{ País } & Usuarios & $\begin{array}{c}\text { Penetración de } \\
\text { Internet en la } \\
\text { población }\end{array}$ \\
\hline 1. Qatar & 2640360 & $99 \%$ \\
\hline $\begin{array}{l}\text { 2. Emiratos Árabes } \\
\text { Unidos }\end{array}$ & 9376171 & $99 \%$ \\
\hline 3. Kuwait & 4100000 & $98 \%$ \\
\hline 4. Bermuda & 60125 & $98 \%$ \\
\hline 5. Bahréin & 1499193 & $98 \%$ \\
\hline 6. Islandia & 329675 & $98 \%$ \\
\hline 7. Noruega & 5222786 & $98 \%$ \\
\hline 8. Andorra & 75366 & $98 \%$ \\
\hline 9. Luxemburgo & 572216 & $98 \%$ \\
\hline 10. Dinamarca & 5571635 & $97 \%$ \\
\hline
\end{tabular}

Fuente: We are Social \& Hootsuite (2018).

De los 213 países analizados en el referido estudio, la penetración más baja correspondió a Corea del Norte $(0.06 \%)$, donde fueron estimados 16 mil usuarios de Internet. Otro dato interesante es la relación de países en los cuales los usuarios destinan el mayor número de horas a Internet (tabla 5). 
Tabla 5: Promedio de horas diarias dedicadas a Internet (enero, 2018).

\begin{tabular}{|l|c|}
\hline \multicolumn{1}{|c|}{ País } & Tiempo \\
\hline 1. Tailandia & 9 horas, 38 minutos \\
\hline 2. Filipinas & 9 horas 29 minutos \\
\hline 3. Brasil & 9 horas 14 minutos \\
\hline 4. Indonesia & 8 horas 51 minutos \\
\hline 5. Sudáfrica & 8 horas 32 minutos \\
\hline 6. Malasia & 8 horas 27 minutos \\
\hline 7. México & 8 horas 17 minutos \\
\hline 8. Argentina & 8 horas 12 minutos \\
\hline 9. Egipto & 8 horas 10 minutos \\
\hline 10. Taiwán & 7 horas 49 minutos \\
\hline
\end{tabular}

Fuente: We are Social \& Hootsuite (2018).

La International Telecommunications Union, ITU (2017) presentó datos mucho más modestos que las dos fuentes que referimos. Según la UIT, al finalizar 2017, el número de usuarios de Internet apenas sería mayor a 3500 mil millones. Además, estimó la penetración mundial de Internet en un 48\%. El crecimiento que registró el sistema digital en 2017 desbordó sus pronósticos.

\section{Penetración de Internet en América}

La IWS (2017) divide al continente americano en cuatro zonas: América del Norte, Sudamérica, Centroamérica y el Caribe. Es importante destacar que la IWS incorporó información relativa a los usuarios de Facebook. El dato es relevante porque representa una primera aproximación a la penetración de las redes sociales en el continente. 
Tabla 6: Población en América, usuarios de Internet y Facebook (junio 2017).

\begin{tabular}{|l|r|r|r|r|}
\hline \multicolumn{1}{|c|}{ Región } & \multicolumn{1}{|c|}{$\begin{array}{c}\text { Población } \\
\text { estimada }\end{array}$} & $\begin{array}{c}\text { Usuarios de } \\
\text { Internet }\end{array}$ & $\begin{array}{c}\text { Penetración de } \\
\text { Internet en la } \\
\text { población }\end{array}$ & $\begin{array}{c}\text { Usuarios de } \\
\text { Facebook }\end{array}$ \\
\hline América del Norte & 363224006 & 320059368 & $88.1 \%$ & 263081200 \\
\hline Sudamérica & 426548298 & 278596721 & $65.3 \%$ & 102760000 \\
\hline Centroamérica & 177249493 & 105771952 & $59.7 \%$ & 10972840 \\
\hline El Caribe & 43806854 & 19900490 & $45.4 \%$ & $\mathbf{6 3 4 0 5 6 5 4 0}$ \\
\hline Total & $\mathbf{1 0 1 0 8 2 8 ~ 6 5 1}$ & $\mathbf{7 2 4 3 2 8 5 3 1}$ & $\mathbf{7 1 . 7 \%}$ & 257200 \\
\hline
\end{tabular}

Fuente: Internet World Stats (2017).

La penetración de Internet en América del Norte $(88.1 \%)$ es superior a los promedios continental $(71.7 \%)$ y mundial $(51.7 \%)$. En cambio, la penetración de Internet en el Caribe (45.4\%) está abajo de ambos promedios. La penetración de Internet en Sudamérica $(65.3 \%)$ y Centroamérica $(59.7 \%)$ es superior al promedio mundial $(51.7 \%)$ pero inferior al continental $(71.7 \%)$. En la tabla 7 presentamos la población estimada en el continente americano, el total de usuarios de Internet, la penetración de Internet en cada país, isla y territorio, así como el total de usuarios de Facebook.

Tabla 7: Usuarios de Internet, Facebook y penetración de Internet en América.

\begin{tabular}{|c|c|c|c|c|c|c|}
\hline Región y país & $\begin{array}{l}\text { Población } \\
\text { estimada }\end{array}$ & Internautas & $\begin{array}{c}\text { Penetración } \\
\text { de Internet en } \\
\text { la población }\end{array}$ & $\begin{array}{c}\text { Usuarios de } \\
\text { Facebook }\end{array}$ & $\begin{array}{c}\text { Penetración } \\
\text { absoluta en } \\
\text { redes sociales }\end{array}$ & $\begin{array}{l}\text { Penetración } \\
\text { relativa }\end{array}$ \\
\hline \multicolumn{7}{|c|}{ América del Norte (junio 2017) } \\
\hline Bermudas & 61352 & 60125 & $98 \%$ & 39000 & $63.5 \%$ & $64.8 \%$ \\
\hline Canadá & 36626083 & 33000381 & $90.1 \%$ & 23000000 & $62.7 \%$ & $69.6 \%$ \\
\hline EE.UU. & 326474013 & 286942362 & $87.9 \%$ & 240000000 & $73.5 \%$ & $83.6 \%$ \\
\hline Groenlandia & 56239 & 52000 & $92.5 \%$ & 38000 & $67.5 \%$ & $73.0 \%$ \\
\hline $\begin{array}{l}\text { San Pedro y } \\
\text { Miquelón }\end{array}$ & 6319 & 4500 & $71.2 \%$ & 4200 & $66.4 \%$ & $93.0 \%$ \\
\hline Total & 363224006 & 320059368 & $88.1 \%$ & 263081200 & $72.4 \%$ & $82.1 \%$ \\
\hline \multicolumn{7}{|c|}{ Centroamérica (junio 2017) } \\
\hline Belice & 374651 & 167020 & $44.6 \%$ & 160000 & $42.7 \%$ & $95.7 \%$ \\
\hline Costa Rica & 4905626 & 4236443 & $86.4 \%$ & 2900000 & $59.1 \%$ & $68.4 \%$ \\
\hline
\end{tabular}




\begin{tabular}{|c|c|c|c|c|c|c|}
\hline El Salvador & 6167147 & 3100000 & $50.3 \%$ & 3100000 & $50.2 \%$ & $100 \%$ \\
\hline Guatemala & 17005497 & 5868597 & $34.5 \%$ & 5300000 & $31.1 \%$ & $90.3 \%$ \\
\hline Honduras & 8304677 & 2700000 & $32.5 \%$ & 2700000 & $32.5 \%$ & $100 \%$ \\
\hline México & 130222815 & 85000000 & $63.5 \%$ & 85000000 & $63.5 \%$ & $100 \%$ \\
\hline Nicaragua & 6217796 & 1900000 & $30.6 \%$ & 1900000 & $30.6 \%$ & $100 \%$ \\
\hline Panamá & 4051284 & 2799892 & $69.1 \%$ & 1700000 & $41.9 \%$ & $60.7 \%$ \\
\hline Total & 177249493 & 105771952 & $59.7 \%$ & 102760000 & $57.9 \%$ & $97.1 \%$ \\
\hline \multicolumn{7}{|c|}{ El Caribe (junio 2016) } \\
\hline Anguila & 14906 & 12557 & $84.2 \%$ & 9000 & $60.3 \%$ & $71.6 \%$ \\
\hline Antigua y Barbuda & 93659 & 68371 & $73 \%$ & 50000 & $53.3 \%$ & $73.1 \%$ \\
\hline Aruba & 104588 & 97832 & $93.5 \%$ & 78000 & $74.6 \%$ & $79.7 \%$ \\
\hline Bahamas & 397164 & 333143 & $83.9 \%$ & 210000 & $52.8 \%$ & $63.0 \%$ \\
\hline Barbados & 285744 & 228717 & $80 \%$ & 160000 & $56.0 \%$ & $69.9 \%$ \\
\hline Bonaire & 25699 & 20956 & $81.5 \%$ & 20 & $0 \%$ & $0 \%$ \\
\hline Cuba & 11390184 & 4415974 & $38.8 \%$ & No reporta & No reporta & No reporta \\
\hline Curazao & 159987 & 138774 & $86.7 \%$ & 80 & $0 \%$ & $0 \%$ \\
\hline Dominica & 73353 & 49147 & $67 \%$ & 39000 & $53.1 \%$ & $79.4 \%$ \\
\hline Granada & 107850 & 60245 & $55,9 \%$ & 56000 & $51.9 \%$ & $92.9 \%$ \\
\hline Guadalupe & 472462 & 220000 & $46.6 \%$ & 220000 & $46.6 \%$ & $100 \%$ \\
\hline Haití & 10983274 & 1343254 & $12.2 \%$ & 1300000 & $11.8 \%$ & $96.9 \%$ \\
\hline Islas Caimán & 61557 & 48630 & $79 \%$ & 45000 & $73.1 \%$ & $92.5 \%$ \\
\hline Islas Turcas y Caicos & 35442 & 25000 & $70.5 \%$ & 25000 & $70.5 \%$ & $100 \%$ \\
\hline Islas Vírgenes (EE. UU.) & 106574 & 63529 & $59.6 \%$ & 21000 & $19.7 \%$ & $33.0 \%$ \\
\hline $\begin{array}{l}\text { Islas Vírgenes } \\
\text { (Reino Unido) }\end{array}$ & 31200 & 14620 & $46.9 \%$ & 4600 & $14.7 \%$ & $31.4 \%$ \\
\hline Jamaica & 2813285 & 1581100 & $56.2 \%$ & 1100000 & $39.1 \%$ & $69.5 \%$ \\
\hline Martinica & 396071 & 303302 & $76.6 \%$ & 170000 & $42.9 \%$ & $56 \%$ \\
\hline Montserrat & 5179 & 2900 & $56 \%$ & 2900 & $56.0 \%$ & $100 \%$ \\
\hline
\end{tabular}




\begin{tabular}{|c|c|c|c|c|c|c|}
\hline Puerto Rico & 3679086 & 3047311 & $82.8 \%$ & 2100000 & $57.0 \%$ & $68.9 \%$ \\
\hline R. Dominicana & 10766564 & 6599904 & $61.3 \%$ & 4500000 & $41.8 \%$ & $68.1 \%$ \\
\hline San Bartolomé (Francia) & 7184 & 1540 & $21.4 \%$ & 20 & $0 \%$ & $0 \%$ \\
\hline San Cristóbal y Nieves & 56780 & 43618 & $76.8 \%$ & 35000 & $61.6 \%$ & $80.2 \%$ \\
\hline Santa Lucía & 187768 & 109370 & $58.2 \%$ & 88000 & $46.8 \%$ & 80.55 \\
\hline San Martín (Francia) & 32125 & 1100 & $3.4 \%$ & 200 & $0 \%$ & $22 \%$ \\
\hline San Martín (Holanda) & 40117 & 20 & $0 \%$ & 20 & $0 \%$ & $100 \%$ \\
\hline $\begin{array}{l}\text { San Vicente y las } \\
\text { Granadinas }\end{array}$ & 109895 & 65984 & $60 \%$ & 59000 & $53.7 \%$ & $89.4 \%$ \\
\hline Trinidad y Tobago & 1369157 & 1003592 & $73.3 \%$ & 700000 & $51.1 \%$ & $69.7 \%$ \\
\hline Total & 43806854 & 19900490 & $45.4 \%$ & 10972840 & $25 \%$ & $55.14 \%$ \\
\hline \multicolumn{7}{|c|}{ Sudamérica (diciembre 2017) } \\
\hline Argentina & 44688864 & 41686960 & $93.1 \%$ & 30000000 & $67.1 \%$ & $71.9 \%$ \\
\hline Bolivia & 11215674 & 7570580 & $67.5 \% \%$ & 6100000 & $54.3 \%$ & $80.5 \%$ \\
\hline Brasil & 210867954 & 149057635 & $70.7 \%$ & 139000000 & $65.9 \%$ & $93.2 \%$ \\
\hline Chile & 18197209 & 14108392 & $77.5 \%$ & 13000000 & $71.4 \%$ & $92.1 \%$ \\
\hline Colombia & 49464683 & 31275567 & $63.2 \%$ & 29000000 & $58.6 \%$ & $92.7 \%$ \\
\hline Ecuador & 16863425 & 13476687 & $79.9 \%$ & 10000000 & $59.3 \%$ & $74.2 \%$ \\
\hline Guayana Francesa & 289763 & 120000 & $41.4 \%$ & 110000 & $37.9 \%$ & $91.6 \%$ \\
\hline Guyana & 782225 & 395007 & $50.5 \%$ & 360000 & $46.0 \%$ & $91.1 \%$ \\
\hline Islas Malvinas (Falkland) & 2922 & 2900 & $99.2 \%$ & $3100^{2}$ & $100 \%$ & $100 \%$ \\
\hline Paraguay & 6896908 & 6177748 & $89.6 \%$ & 3300000 & $47.8 \%$ & $53.4 \%$ \\
\hline Perú & 35551815 & 22000000 & $67.6 \%$ & 20000000 & $56.2 \%$ & $91 \%$ \\
\hline Surinam & 568301 & 340000 & $59.8 \%$ & 310000 & $54.5 \%$ & $91.1 \%$ \\
\hline Uruguay & 3469551 & 3059727 & $88.2 \%$ & 2400000 & $69.1 \%$ & $78.4 \%$ \\
\hline Venezuela & 32381221 & 17178743 & $53.1 \%$ & 13000000 & $40.1 \%$ & $75.6 \%$ \\
\hline Total (diciembre 2017) & 428240515 & 306349946 & $71.5 \%$ & 266583100 & $62.1 \%$ & $86.9 \%$ \\
\hline
\end{tabular}

Fuente: Internet World Stats (2017).

2 Según IWS (2017), el número de usuarios de Facebook supera a la población total. 
La penetración de Internet en cada uno de los territorios y naciones en América del Norte es superior al promedio mundial (51.7\%): Bermudas (98\%), Canadá (90.1\%), Estados Unidos (89.7\%), Groenlandia (92.5\%), San Pedro y Miquelón (71.2\%). Únicamente en San Pedro y Miquelón la penetración de Internet en la población se ubica abajo del promedio continental $(71.2 \%)$.

En Centroamérica, Costa Rica presenta la más alta penetración de Internet $(86.4 \%)$, superior al promedio continental (71.7\%) y mundial (51.7\%). En Panamá (69.1\%), y México (65.3\%) es superior al promedio mundial pero inferior al promedio continental. En Belice (44.6\%), El Salvador (50.3\%), Guatemala (34.5\%), Honduras (32.5\%), y Nicaragua $(30.6 \%)$, el país más rezagado en esa región, la penetración de Internet se ubica debajo de ambos promedios.

Aruba presentó la penetración más alta de Internet en la población (93.5\%). Además de Aruba, la penetración de Internet en las siguientes islas fue superior al promedio mundial (51.7\%) y al promedio continental (71.7\%): Curazao (86.7\%), Anguila (84.2\%), Bahamas (83.9\%), Puerto Rico (82.8\%), Bonaire (81.5\%) Barbados (80\%), Islas Caimán (79\%), San Cristóbal y Nieves (76.8\%), Martinica (76.6\%), Trinidad y Tobago (73.3\%) y Antigua y Barbuda $(73 \%)^{3}$.

En las siguientes islas, la penetración de Internet fue superior al promedio mundial pero inferior al continental: Islas Turcas y Caicos (70.5\%) Dominica (67\%), República Dominicana (61.3\%), San Vicente y Granadinas (60\%), Islas Vírgenes (Estados Unidos, $59.6 \%$ ), Santa Lucía (58.2\%), Jamaica (56.2\%), Montserrat (56\%) y Granada (55.9\%). Por debajo de ambos promedios fueron ubicadas las siguientes islas: Islas Vírgenes (Reino Unido, 46.9\%), Guadalupe (46.6\%), Cuba (38.8\%), San Bartolomé (21.4\%), Haití (12.2\%), San Martín (Francia, 3.4\%), San Martín (Holanda, 0\%).

Por lo que respecta a Sudamérica, en las Islas Malvinas se registró la más alta penetración de Internet (99.2\%). En estas naciones la penetración de Internet es superior al promedio mundial (54.4\%) y al promedio continental (71.5\%): Argentina (93.1\%), Chile (77.5\%), Ecuador $(79.9 \%)$, Paraguay $(89.6 \%)^{4}$ y Uruguay $(88.2 \%)$. Por encima del promedio mundial, pero debajo del continental se encuentran: Bolivia $(67.6 \%)$, Brasil $(70.7 \%)$, Colombia (63.2\%) y Perú (67.6\%). Debajo de ambos promedios: Guyana (50.5\%), Guyana Francesa (41.4\%). Surinam (59.8\%) y Venezuela (53.1\%).

3 Es importante tener presente que, al momento de escribir el texto, la información más reciente de la IWS (2017) sobre la penetración de Internet en las islas caribeñas correspondía a junio de 2016.

4 Es importante destacar que, de acuerdo con el estudio de We are social \& Hootsuite (2018), Paraguay fue ubicado en la séptima posición entre los 10 países que presentaron el mayor crecimiento en la penetración de Internet en el periodo enero 2017- enero 2018. 


\section{Las redes sociales}

En este apartado centramos nuestra atención en las redes sociales, complejo subsistema en la web que en años recientes ha experimentado una formidable expansión hasta convertirse en el territorio más visible, cotizado y codiciado en Internet. Para ello recurrimos a información de la IWS (2017) y a We are Social \& Hootsuite $(2017,2018)$ para reconocer la penetración mundial de las redes sociales, las cuales, desde la óptica de la "ecología de los medios", admiten ser comprendidas como venas y arterias del organismo social.

\subsection{Penetración de las redes sociales}

De acuerdo con We are social \& Hootsuite (2018), en enero de 2018 fueron estimados en 3 196000000 los usuarios de redes sociales. La penetración absoluta de las redes sociales fue establecida en $42 \%{ }^{6}$. De cada 10 personas en el mundo, 4 son usuarios de alguna o algunas redes sociales. En el periodo comprendido entre enero de 2017 y enero de 2018, el incremento observado en la penetración absoluta de las redes sociales fue estimado en 13\%. Además, en ese periodo fueron estimados 362 millones de nuevos usuarios de las redes sociales 7.

Tabla 8: Total de usuarios activos de redes sociales y en conexiones móviles.

\begin{tabular}{|l|r|r|r|r|r|r|}
\hline \multicolumn{1}{|c|}{ Región } & \multicolumn{1}{|c|}{$\begin{array}{c}\text { Conexiones } \\
\text { móviles }\end{array}$} & $\begin{array}{c}\text { Penetración } \\
\text { absoluta }\end{array}$ & $\begin{array}{c}\text { Usuarios } \\
\text { activos de redes } \\
\text { sociales }\end{array}$ & $\begin{array}{c}\text { Penetración } \\
\text { absoluta }\end{array}$ & $\begin{array}{c}\text { Usuarios móviles } \\
\text { activos de redes } \\
\text { sociales }\end{array}$ & $\begin{array}{c}\text { Penetración } \\
\text { absoluta }\end{array}$ \\
\hline África & 1040000000 & $82 \%$ & 191000000 & $15 \%$ & 172000000 & $14 \%$ \\
\hline América & 1,070000000 & $106 \%$ & 648000000 & $64 \%$ & 581000000 & $57 \%$ \\
\hline Asia-Pacífico & 4318000000 & $102 \%$ & 1779000000 & $42 \%$ & 1713000000 & $41 \%$ \\
\hline Europa & 1106000000 & $131 \%$ & 448000000 & $53 \%$ & 376000000 & $45 \%$ \\
\hline $\begin{array}{l}\text { Medio } \\
\text { Oriente }\end{array}$ & 323000000 & $128 \%$ & 130000000 & $52 \%$ & 115000000 & $46 \%$ \\
\hline Totales & $\mathbf{7 8 5 7 0 0 0 ~ 0 0 0}$ & $\mathbf{1 0 9 . 8 \%}$ & $\mathbf{3 1 9 6 0 0 0}$ & $\mathbf{4 2 \%}$ & $\mathbf{2 9 5 8 0 0 0}$ & $\mathbf{3 9 \%}$ \\
\hline
\end{tabular}

Fuente: We are Social \& Hootsuite (2018).

5 La ecología de los medios o Media Ecology es una compleja metadisciplina que pretende explicar el impacto de las tecnologías en la cultura de las sociedades a través de la historia. El punto de partida de la ecología de los medios es el pensamiento de Marshall McLuhan.

6 Partiendo de esos datos es posible determinar la penetración relativa de las redes sociales, la cual corresponde al porcentaje que representan los usuarios de las redes sociales respecto del total de usuarios de Internet. Si consideramos 4201000000 usuarios de Internet en el mundo (dato asentado en el estudio Digital in 2018. Global Overview) y 3196000000 de usuarios de redes sociales en el mundo, la penetración relativa de las redes sociales en el mundo es del 79.48\%. Esto quiere decir que de cada 10 usuarios de Internet, 8 son usuarios de redes sociales.

7 Las penetraciones absolutas (tercera, quinta y séptima columnas) están determinadas con base en la población total. 
Según la tabla 8, Asia-Pacífico presenta el mayor número de conexiones móviles (4 318 000 000). Sin embargo, en Europa se registra la mayor penetración (131\%). En todos los bloques la penetración absoluta de las conexiones es superior a la población estimada, con excepción de África (82\%). En Asia Pacífico se presenta el mayor número de usuarios activos de redes sociales (1 779000 000) y en África, el menor número de usuarios (191 000 000). La mayor penetración absoluta de usuarios activos de redes sociales se presenta en América (64\%) y la menor, en África (15\%).

Con respecto a los usuarios móviles de redes sociales, sorprende advertir que de cada 10 personas en el mundo, 4 son usuarios móviles de redes sociales (los 2958000000 usuarios móviles de redes sociales representan 39\% de la población mundial). Asia-Pacífico presenta el mayor número de usuarios móviles de redes sociales (1 713000 000). En América se registra la mayor penetración (57\%). En el periodo comprendido entre enero de 2017 y enero de 2018, fueron estimados 360 millones de nuevos usuarios móviles de redes sociales en el mundo. En la tabla 9 podremos apreciar la penetración de las redes sociales por regiones.

Tabla 9: Penetración de redes sociales por regiones (enero 2018).

\begin{tabular}{|c|c|c|}
\hline Región & Millones de usuarios & Penetración absoluta \\
\hline Este de Asia & 1053 & $64 \%$ \\
\hline Sur de Asia & 375 & $20 \%$ \\
\hline Sureste de Asia & 360 & $55 \%$ \\
\hline Sudamérica & 270 & $63 \%$ \\
\hline América del Norte & 255 & $70 \%$ \\
\hline Oeste de Asia & 148 & $55 \%$ \\
\hline Europa del Este & 131 & $45 \%$ \\
\hline Centroamérica & 106 & $59 \%$ \\
\hline Europa del Oeste & 104 & $54 \%$ \\
\hline Norte de África & 90 & $38 \%$ \\
\hline Sur de Europa & 86 & $56 \%$ \\
\hline Norte de Europa & 66 & $69 \%$ \\
\hline Oeste de África & 41 & $11 \%$ \\
\hline Este de África & 30 & $7 \%$ \\
\hline Oceanía & 23 & $55 \%$ \\
\hline
\end{tabular}




\begin{tabular}{|l|c|c|}
\hline África del Sur & 31 & $20 \%$ \\
\hline Caribe & 18 & $40 \%$ \\
\hline África Central & 10 & $6 \%$ \\
\hline Asia Central & 9 & $12 \%$ \\
\hline
\end{tabular}

Fuente: We are Social \& Hootsuite (2018).

En el Este de Asia se concentra el mayor número de usuarios de redes sociales (1,053 millones). Sin embargo, la penetración más elevada se presenta en América del Norte (70\%), y la más baja en África Central (6\%). We are Social \& Hootsuite (2018) además ofrece información sobre los países que presentan la más alta penetración de redes sociales (tabla 10).

Tabla 10: Países que presentan la más alta penetración de redes sociales.

\begin{tabular}{|l|r|c|}
\hline \multicolumn{1}{|c|}{ País } & \multicolumn{1}{|c|}{ Usuarios } & $\begin{array}{c}\text { Penetración de Internet } \\
\text { en la población }\end{array}$ \\
\hline 1. Qatar & 2640360 & $99 \%$ \\
\hline 2. Emiratos Árabes Unidos & 9376000 & $99 \%$ \\
\hline 3. Kuwait & 4100000 & $98 \%$ \\
\hline 4. Brunéi & 60125 & $98 \%$ \\
\hline 5. Aruba & 100000 & $95 \%$ \\
\hline 6. Bahréin & 1400000 & $92 \%$ \\
\hline 7. Islas Caimán & 54000 & $87 \%$ \\
\hline 8. Corea del Sur & 43044000 & $84 \%$ \\
\hline 9. Singapur & 4800000 & $83 \%$ \\
\hline 10. Malta & 360000 & $83 \%$ \\
\hline
\end{tabular}

Fuente: We are Social \& Hootsuite (2018).

We are Social \& Hootsuite (2018) también incluyó la relación de países en los cuales los internautas dedican mayor cantidad de tiempo a las redes sociales (tabla 11). Tres países en el continente americano aparecen en el top ten: Brasil, en segundo lugar; Argentina, en quinto; y México, en séptima posición. 
Tabla 11: Promedio de horas dedicadas a Internet (enero, 2018).

\begin{tabular}{|c|c|}
\hline País & Tiempo \\
\hline 1. Filipinas & 3 horas 57 minutos \\
\hline 2. Brasil & 3 horas 39 minutos \\
\hline 3. Indonesia & 3 horas 23 minutos \\
\hline 4. Tailandia & 3 horas 10 minutos \\
\hline 5. Argentina & 3 horas 9 minutos \\
\hline 6. Egipto & 3 horas 9 minutos \\
\hline 7. México & 3 horas 7 minutos \\
\hline 8. Nigeria & 3 horas 2 minutos \\
\hline 9. Malasia & 3 horas \\
\hline 10. Emiratos Árabes Unidos & 2 horas 56 minutos \\
\hline
\end{tabular}

Fuente: We are Social \& Hootsuite (2018).

De acuerdo con lo detallado en We are social \& Hootsuite (2018), estas son las principales redes sociales y plataformas de mensajería en el mundo, así como los usuarios mensuales estimados (tabla 12).

Tabla 12: Principales redes sociales y plataformas, con usuarios estimados (mensual).

\begin{tabular}{|l|l|r|}
\hline \multirow{5}{*}{ Redes sociales } & Red social o plataforma & \multicolumn{1}{|c|}{$\begin{array}{c}\text { Usuarios } \\
\text { (en millones) }\end{array}$} \\
\hline & Facebook & 2167 \\
\cline { 2 - 3 } & YouTube & 1500 \\
\cline { 2 - 3 } & Instagram & 800 \\
\cline { 2 - 3 } & Tumbrl & 794 \\
\cline { 2 - 3 } & QZone & 768 \\
\cline { 2 - 3 } & Sina Weibo & 1300 \\
\hline \multirow{5}{*}{$\begin{array}{l}\text { Plataforma, servicios de } \\
\text { mensajería, chat App, VOIP }\end{array}$} & WhatsApp & 1300 \\
\cline { 2 - 3 } & Facebook Messenger & 980 \\
\cline { 2 - 3 } & Wechat & 843 \\
\cline { 2 - 3 } & QQ & \\
\hline
\end{tabular}

Fuente: We are Social \& Hootsuite (2018). 
Twitter (considerada como una de las redes sociales más influyentes en la opinión pública internacional), fue ubicada en la undécima posición, con 330 millones de usuarios. Cabe destacar que en el periodo de enero 2017 - enero 2018, Facebook experimentó un crecimiento estimado en $15 \%$ en el número de usuarios. Del total de usuarios de esa red social (2167 millones), se estima que $88 \%$ accede y usa esta red social a través de dispositivos móviles (principalmente por el teléfono celular). Además, del total de usuarios de Facebook, 44\% declararon ser mujeres y $56 \%$ hombres.

Tabla 13: Países con el mayor número de usuarios de Facebook.

\begin{tabular}{|l|r|c|}
\hline & Usuarios & Porcentaje del total \\
\hline 1. India & 250000000 & $12 \%$ \\
\hline 2. Estados Unidos & 230000000 & $11 \%$ \\
\hline 3. Brasil & 130000000 & $6 \%$ \\
\hline 4. Indonesia & 130000000 & $6 \%$ \\
\hline 5. México & 83000000 & $4 \%$ \\
\hline 6. Filipinas & 67000000 & $3 \%$ \\
\hline 7. Vietnam & 55000000 & $3 \%$ \\
\hline 8. Tailandia & 51000000 & $2 \%$ \\
\hline 9. Turquía & 51000000 & $2 \%$ \\
\hline 10. Reino Unido & 44000000 & $2 \%$ \\
\hline
\end{tabular}

Fuente: We are Social \& Hootsuite (2018).

En la relación de ciudades con el mayor número de usuarios de Facebook figuran las dos grandes urbes latinoamericanas: la Ciudad de México, en quinta posición, con 14 millones de usuarios, y Sao Paolo, en octavo sitio, con 13 millones de usuarios. La ciudad que concentra el mayor número de usuarios de Facebook es Bangkok, con 22 millones.

\subsection{Usuarios de Facebook en América}

Sobre la base de la información que ofrece la IWS (2017) sobre población estimada, usuarios de Internet, penetración de Internet en la población y usuarios de Facebook en América ${ }^{8}$, determinamos la penetración absoluta y relativa de las redes sociales por regiones (tabla 14).

8 A partir de la penetración de Facebook suele determinarse la penetración de las redes sociales. La razón es lógica: Facebook es la red social que cuenta con el mayor número de usuarios en el mundo. 
Tabla 14: Usuarios de Internet y Facebook en América (junio 2017).

\begin{tabular}{|l|r|r|r|r|r|}
\hline \multicolumn{1}{|c|}{ Región } & \multicolumn{1}{|c|}{$\begin{array}{c}\text { Población } \\
\text { estimada }\end{array}$} & \multicolumn{1}{c|}{$\begin{array}{c}\text { Usuarios de } \\
\text { Internet }\end{array}$} & \multicolumn{1}{c|}{$\begin{array}{c}\text { Usuarios de } \\
\text { Facebook }\end{array}$} & $\begin{array}{c}\text { Penetración } \\
\text { absoluta } \\
\text { Facebook }\end{array}$ & $\begin{array}{c}\text { Penetración } \\
\text { relativa } \\
\text { Facebook }\end{array}$ \\
\hline América del Norte & 363224006 & 320059368 & 263081200 & $72.4 \%$ & $82.1 \%$ \\
\hline Sudamérica & 426548298 & 278596721 & 257242500 & $47.4 \%$ & $92.4 \%$ \\
\hline Centroamérica & 177249493 & 105771952 & 102760000 & $57.6 \%$ & 97.1 \\
\hline El Caribe & 43806854 & 19900490 & 10972840 & $25.3 \%$ & $57.3 \%$ \\
\hline Total & $\mathbf{1 0 1 0 ~ 8 2 8 ~ 6 5 1}$ & $\mathbf{7 2 4} \mathbf{3 2 8 5 3 1}$ & $\mathbf{6 3 4 0 5 6 5 4 0}$ & $\mathbf{6 2 . 7 \%}$ & $\mathbf{8 7 . 5 \%}$ \\
\hline
\end{tabular}

Fuente: Elaboración propia, basada en Internet World Stats (2017).

El promedio de penetración absoluta de Facebook en el continente americano es del 62.7\%. El porcentaje más alto se presenta en América del Norte (72.4\%). El promedio es inferior en las regiones restantes (Sudamérica, 47.4\%; Centroamérica, 57.6\% y El Caribe, 25.3\%).

El promedio de penetración relativa de Facebook en el continente es del 87.5\%. Dos regiones presentan una penetración relativa más alta que la registrada en el promedio continental: Sudamérica (92.4\%) y Centroamérica (97.1\%). Debajo del promedio continental se encuentran Norteamérica (82.1\%) y el Caribe (57.3\%).

Como se puede apreciar en la tabla 7, en América del Norte, el promedio de la penetración absoluta de redes sociales es $72.4 \%$ (la más alta en el continente), y la relativa fue establecida en $82.1 \%$. Superior al promedio absoluto únicamente se encuentran los Estados Unidos $(73.5 \%)$, nación que además también se ubica arriba del promedio relativo en la región $(82.1 \%)$, el promedio relativo fue estimado en $83.6 \%$ en la Unión Americana. Además de los Estados Unidos, San Pedro y Miquelon (93\%) se ubica arriba del promedio relativo en la región.

En Centroamérica, el promedio de la penetración absoluta de redes sociales es $57.9 \%$, y la penetración relativa $(97.1 \%)$, la más alta en el continente. Esto se debe a que en cuatro países (El Salvador, Honduras, México y Nicaragua) la penetración relativa es 100\% (todos los usuarios de Internet son usuarios de redes sociales. En cambio, Panamá y Costa Rica (países que presentan la más alta penetración de Internet en esta zona) son las naciones que presentan la penetración relativa más baja (60.7\% y $68.4 \%$, respectivamente). En México, la penetración absoluta es la más alta en la región (63.5\%). Además de México, en Costa Rica la penetración absoluta de redes sociales (59.1\%) se ubica arriba del promedio regional (57.1\%).

En cuanto a El Caribe, el promedio de la penetración absoluta de redes sociales $(25 \%)$ es el más bajo en el continente, así como la relativa (55.14\%). Por encima del promedio regional, tanto absoluto como relativo, se encuentran: Anguila, Antigua y Barbuda, Aruba, Bahamas, Barbados, Dominica, Granada, Guadalupe, Islas Caimán, Islas Turcas 
y Caicos, Islas Vírgenes (Estados Unidos), Jamaica, Martinica, Montserrat, Puerto Rico, República Dominicana, San Cristóbal y Nieves, Santa Lucía, San Vicente y Granadinas, Trinidad y Tobago. Arriba del promedio relativo (55.14\%): Haití (96.9\%) y San Martín, Holanda (100\%). Debajo de ambos promedios se ubican las siguientes islas: Bonaire, Curazao, Islas Vírgenes (Estados Unidos), Islas Vírgenes (Reino Unido), San Bartolomé y San Martín (Francia).

En Sudamérica se concentra el mayor número de usuarios de redes sociales en el continente (266 583 100). El promedio de la penetración absoluta de redes sociales asciende a $62.1 \%$ y la relativa es de $86.9 \%$. Por arriba de ambos promedios se encuentran: Argentina, Brasil, Chile y las Islas Malvinas (Falkland). Abajo del promedio absoluto: Colombia (58.6\%), Guyana Francesa (37.9\%), Guyana (46\%), Perú (56.2\%) y Surinam (54.5\%). Abajo del promedio relativo: Uruguay (78.4\%). Debajo de ambos promedios se encuentran: Bolivia, Ecuador, Paraguay y Venezuela.

\section{La importancia que el Foro Económico Mundial concede al uso de las redes sociales}

The Global Information Technology Report (2016) fue resultado de un proyecto especial que realizó el Foro Económico Mundial, el INSEAD ${ }^{9}$ y la Universidad de Cornell (Estados Unidos) para el Equipo Global de Competitividad y Riesgos del Foro Económico Mundial y el Programa de Alianzas Industriales para Tecnologías de la Información y la Comunicación. El propósito del estudio es identificar áreas de prioridad para aprovechar al máximo las tecnologías de información y comunicaciones (TIC) para el desarrollo socioeconómico. Las premisas que fundamentan el mencionado reporte son:

a) un entorno regulatorio y empresarial de alta calidad es fundamental para aprovechar plenamente las TIC y generar impacto; b) la disponibilidad de TIC, medida por la asequibilidad, las habilidades y la infraestructura de las TIC, es una condición previa para generar impacto; c) el pleno aprovechamiento de las TIC requiere un esfuerzo en toda la sociedad: el gobierno, el sector empresarial y la población en general tienen un papel fundamental que desempeñar; d) El uso de las TIC no debe ser un fin en sí mismo. El impacto que las TIC realmente tienen en la economía y la sociedad es lo que finalmente importa; $e$ ) el conjunto de controladores, el entorno, la preparación y el uso, interactúan, coevolucionan y se refuerzan mutuamente para formar un círculo virtuoso; y $f$ ) el marco de preparación en red debe proporcionar una orientación política clara (World Economic Forum, 2016, p. 13).

9 Es una escuela de negocios y un centro de investigación con campus en Francia (Fontainebleau), Singapur y Abu Dabi. El nombre oficial es Institut Européen d'Administration des Affaires. 
El estudio comprende cuatro categorías principales (subíndices): "ambiente", "preparación", "uso" e "impacto". De los cuatro subíndices se desprenden 10 subcategorías (pilares) y 53 indicadores. El subíndice "ambiente" cuenta con dos subcategorías: a) entorno político y normativo (9 indicadores) y b) entorno empresarial y de innovación (9 indicadores). El subíndice "preparación" tiene tres subcategorías: $c$ ) infraestructura (4 indicadores), d) asequibilidad (3 indicadores) y e) habilidades (4 indicadores). El subíndice "uso" comprende 3 subcategorías: $f$ ) uso individual (7 indicadores), g) uso comercial (6 indicadores), h) uso del gobierno (3 indicadores). Por último, el subíndice "impacto" incluye dos subcategorías: $i$ ) impactos económicos (4 indicadores) y j) impactos sociales (4 indicadores). La puntuación global se determina sobre la base de los puntajes que arrojan cada uno de los indicadores para obtener puntajes de pilar, que luego se combinan para obtener los puntajes del subíndice. Los puntajes de los subíndices permiten determinar el puntaje de cada país ${ }^{10}$.

El indicador relativo al empleo de las redes sociales (use of virtual social networks) fue incorporado en el estudio correspondiente al 2010-2011. En el estudio más reciente, el referido indicador fue considerado en el grupo de 7 indicadores que forman parte de la subcategoría "uso individual". Los seis indicadores restantes de la subcategoría uso individual son: a) suscripciones a teléfonos móviles por cada 100 habitantes; $b$ ) porcentaje de personas que usan Internet; c) porcentaje de hogares con computadoras; $d$ ) porcentaje de hogares con acceso a Internet; e) suscripciones a Internet de banda ancha fija por cada 100 habitantes y f) suscripciones a Internet de banda ancha móvil por cada 100 habitantes.

En la reciente edición del estudio, 24 países en América fueron analizados: Argentina, Bolivia, Brasil, Canadá, Chile, Colombia, Costa Rica, Ecuador, El Salvador, Estados Unidos, Guatemala, Guyana, Haití, Jamaica, México, Nicaragua, Panamá, Paraguay, Perú, República Dominicana, Trinidad y Tobago, Uruguay, Venezuela. Las mejores evaluaciones (en general) fueron asignadas a Estados Unidos (ubicado en la quinta posición mundial), Canadá (14), Chile (38), Uruguay (43) y Costa Rica (44). Por lo que respecta al uso de redes sociales, las mejores posiciones correspondieron a Estados Unidos (3), Canadá (18), Chile (37), Panamá (39) y Costa Rica (44).

La tabla 15 consigna las evaluaciones que fueron concedidas en el indicador uso de redes sociodigitales a las naciones del continente americano que fueron analizadas en este estudio.

10 Las principales fuentes de información son la International Telecommunications Union, la ONU, la UNESCO y el Banco Mundial. Además, se aplica una encuesta a ejecutivos de negocios. 
Tabla 15: Evaluaciones a las naciones del continente americano.

\begin{tabular}{|c|c|c|c|c|}
\hline País & $\begin{array}{c}\text { Posición en el } \\
\text { estudio }\end{array}$ & $\begin{array}{c}\text { Posición por el } \\
\text { porcentaje de } \\
\text { usuarios de Internet }\end{array}$ & $\begin{array}{c}\text { Posición en la } \\
\text { subcategoría uso } \\
\text { individual }\end{array}$ & $\begin{array}{c}\text { Uso de redes } \\
\text { sociodigitales }\end{array}$ \\
\hline Argentina & 89 & 48 & 53 & 53 \\
\hline Bolivia & 111 & 91 & 108 & 132 \\
\hline Brasil & 72 & 58 & 57 & 46 \\
\hline Canadá & 14 & 14 & 30 & 18 \\
\hline Chile & 38 & 36 & 52 & 37 \\
\hline Colombia & 68 & 66 & 71 & 89 \\
\hline Costa Rica & 44 & 69 & 55 & 55 \\
\hline Ecuador & 82 & 82 & 87 & 114 \\
\hline E1 Salvador & 93 & 96 & 91 & 79 \\
\hline Estados Unidos & 5 & 13 & 79 & 3 \\
\hline Guatemala & 103 & 101 & 100 & 71 \\
\hline Guyana & 100 & 92 & 105 & 94 \\
\hline Haití & 137 & 120 & 136 & 124 \\
\hline Honduras & 94 & 103 & 104 & 65 \\
\hline Jamaica & 83 & 86 & 86 & 75 \\
\hline México & 76 & 78 & 111 & 91 \\
\hline Nicaragua & 131 & 110 & 67 & 125 \\
\hline Panamá & 55 & 77 & 72 & 39 \\
\hline Paraguay & 105 & 82 & 96 & 102 \\
\hline Perú & 90 & 88 & 93 & 103 \\
\hline República Dominicana & 98 & 68 & 95 & 83 \\
\hline Trinidad y Tobago & 67 & 47 & 59 & 33 \\
\hline Uruguay & 43 & 53 & 44 & 64 \\
\hline Venezuela & 108 & 59 & 74 & 61 \\
\hline
\end{tabular}

Fuente: World Economic Forum (2016). 


\section{Conclusiones}

Los datos reportados por la International Telecommunications Union (2017), Internet World Stats (2017), World Economic Forum (2016) y We are Social \& Hootsuite (2017, 2018) sobre la penetración de Internet en el mundo, reflejan que cada vez hay un mayor número de usuarios, de tal manera que ya superan la mitad de la población global. Ello representa así una excelente oportunidad no sólo para los negocios sino para la inclusión digital. Aun así, siguen existiendo las brechas digitales por regiones. Aunque, el acceso a Internet no está generalizado en todas las partes del mundo, existen tendencias que comienzan a invertirse.

América del Norte y Europa presentan promedios superiores a la media mundial, mientras que algunos países han experimentado un notable incremento de usuarios. Por ejemplo, en África central se está experimentando un importante crecimiento en cuanto acceso de su población a Internet, superior a la media mundial.

Hay que destacar que más de la mitad de la población que se conecta a Internet lo hace a través de dispositivos móviles y decrecen los usuarios que prefieren navegar desde una computadora de escritorio, laptop y tableta. Precisamente, el aumento del acceso a Internet y redes sociales está muy vinculado al uso de smartphones y planes de datos, ambos cada vez más asequibles. Además, los teléfonos celulares son el dispositivo principal utilizado por nuevos usuarios de internet para acceder a redes sociales, siendo la actividad en redes sociales la más preponderante en las conexiones móviles.

El aumento en el número de usuarios de internet y usuarios de las redes sociales viene acompañado también por mayor cantidad de tiempo en línea. Los estudios demuestran que más de la mitad del tiempo que permanecemos despiertos, estamos conectados a Internet. Facebook sigue siendo la red social más popular y con un crecimiento sostenido de usuarios. Además, cuatro de las siete redes sociales más importantes pertenecen a Mark Zuckerberg (Facebook, WhatsApp, Messenger e Instagram). Precisamente, Instagram es la red social que ha experimentado un crecimiento espectacular, logrando duplicar el número de usuarios en solo dos años.

Si bien los estudios todavía no reportan estadísticas sobre Google Home, Amazon Echo (sistemas basados en interacción por voz) ni sobre Siri, Alexa o Google Now (asistentes virtuales), su irrupción marcan un futuro en el que los usuarios realizarán casi cualquier acción sobre su dispositivo móvil sin utilizar las manos, haciendo presagiar que el audio online va a ser un formato cada vez más empleado. Así lo ha entendido no solo Facebook (con Audio Live para transmitir audio en directo), sino también Amazon con Amazon Music y Spotify.

Los recientes estudios muestran patrones de comportamiento en el ámbito digital, donde las tecnologías con reconocimiento de voz, el dominio del contenido audiovisual en las redes sociales, entre otras tendencias, llevan a una mejor experiencia digital para el consumidor. Esto hace repensar las estrategias de las economías más avanzadas y emergentes del mundo. 


\section{Referencias}

International Telecommunications Union, ITU (2017). Measuring the Information Society Report. Volumen 1. Recuperado de https:/www.itu.int/en/ITU-D/ Statistics/Documents/publications/misr2017/MISR2017_Volume1.pdf

Internet World Stats, IWS (2017). World Internet Users and 2017 Population Stats. Recuperado de http://www.internetworldstats.com/stats.htm

Statista (2017a). Countries with the most Facebook users as of April 2017. Recuperado de https://www.statista.com/statistics/268136/top-15-countries-based-on-numberof-facebook-users/

Statista (2017b). Most famous social network sites 2017. Recuperado de https://www. statista.com/statistics/272014/global-social-networks-ranked-by-number-ofusers/

We are social \& Hootsuite (2017). Digital in 2017. Global Overview. A collection of Internet, Social Media and Mobile Data from around the World. Recuperado de https://wearesocial.com/special-reports/digital-in-2017-global-overview

We are social \& Hootsuite (2018). Digital in 2018. Global Overview. A collection of Internet, Social Media and Mobile Data from around the World. Recuperado de https://digitalreport.wearesocial.com/

World Economic Forum (2016). The Global Information Technology Report 2016. Recuperado de https://www.weforum.org/reports/the-global-informationtechnology-report-2016 